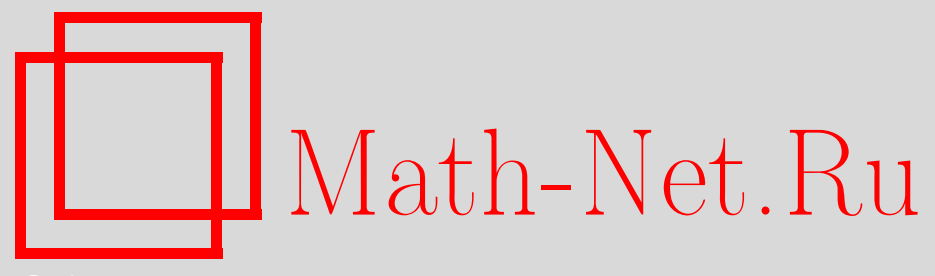

В. Н. Маклаков, Сходимость матричного метода численного интегрирования краевых задач для линейных неоднородных обыкновенных дифференциальных уравнений второго порядка с переменными коэффициентами, Вестн. Сам. гос. техн. ун-та. Сер. Физ.-мат. науки, 2015, номер 3, 559577

DOI: https://doi.org/10.14498/vsgtu1426

Использование Общероссийского математического портала MathNet.Ru подразумевает, что вы прочитали и согласны с пользовательским соглашением

http://www. mathnet.ru/rus/agreement

Параметры загрузки:

IP : 54.197 .130 .99

26 апреля 2023 г., $14: 24: 10$

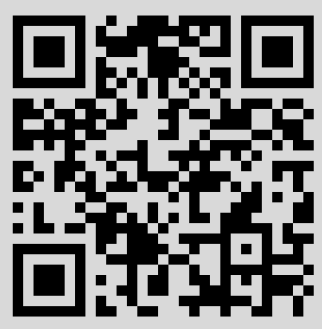




\title{
СХОДИМОСТЬ МАТРИЧНОГО МЕТОДА ЧИСЛЕННОГО ИНТЕГРИРОВАНИЯ КРАЕВЫХ ЗАДАЧ ДЛЯ ЛИНЕЙНЫХ НЕОДНОРОДНЫХ ОБЫКНОВЕННЫХ ДИФФЕРЕНЦИАЛЬНЫХ УРАВНЕНИЙ ВТОРОГО ПОРЯДКА С ПЕРЕМЕННЫМИ КОЭФФИЦИЕНТАМИ
}

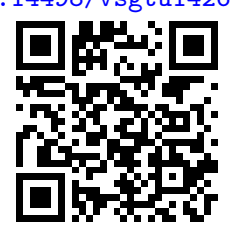

\section{В. Н. Маклаков}

Самарский государственный технический университет, Россия, 443100, Самара, ул. Молодогвардейская, 244.

\begin{abstract}
Аннотация
Исследованы вопросы устойчивости и сходимости предложенного ранее матричного метода численного интегрирования краевых задач с граничными условиями первого, второго и третьего рода для линейных неоднородных обыкновенных дифференциальных уравнений второго порядка с переменными коэффициентами. Использование многочленов Тейлора произвольных степеней позволило повысить порядок аппроксимации метода до произвольного натурального числа и отказаться от аппроксимации производных конечными разностями. При выборе второй степени многочлена Тейлора уравнения метода совпали с известными уравнениями традиционного метода численного интегрирования краевых задач, в котором производные аппроксимированы конечными разностями. Показано, что достаточный критерий устойчивости при использовании в методе многочленов Тейлора степени три и выше совпадает с достаточным критерием устойчивости традиционного метода сеток для численного интегрирования краевых задач с граничными условиями первого, второго и третьего рода. Теоретически установлено, что скорость сходимости матричного метода интегрирования краевых задач с граничными условиями первого рода пропорциональна степени используемого многочлена Тейлора в случае, когда эта степень является чётной, и пропорциональна числу, меньшему степени на единицу, когда эта степень является нечётной; при интегрировании краевых задач с граничными условиями второго и третьего рода скорость сходимости метода пропорциональна степени используемого многочлена Тейлора независимо от её чётности и меньше неё на единицу. Полученные теоретические результаты подтверждены численным экспериментом.
\end{abstract}

Ключевые слова: обыкновенные дифференциальные уравнения, краевые задачи, порядок аппроксимации, устойчивость, сходимость, численные методы, многочлены Тейлора.

doi: http://dx.doi.org/10.14498/vsgtu1426

(C) 2015 Самарский государственный технический университет.

\section{Образец для цитирования}

Маклаков В. Н. Сходимость матричного метода численного интегрирования краевых задач для линейных неоднородных обыкновенных дифференциальных уравнений второго порядка с переменными коэффициентами // Вестн. Сам. гос. техн. ун-та. Сер. Физ.мат. науки, 2015. Т. 19, № 3. С. 559-577. doi: 10.14498/vsgtu1426.

Сведения об авторе

Владимир Николаевич Маклаков (к.ф.-м.н., доц.; makvo63@уandex.ru), доцент, каф. высшей математики и прикладной информатики. 
В классическом методе численного интегрирования краевых задач для неоднородных обыкновенных дифференциальных уравнений второго порядка (ОДУ2) с переменными коэффициентами

$$
u^{\prime \prime}+p(x) u^{\prime}+q(x) u=f(x), \quad u_{0}=\widetilde{u}_{0}, \quad u_{n}=\widetilde{u}_{n},
$$

где $p(x), q(x), f(x)$ - заданные функции, дифференцируемые нужное число раз, $[a, b]$ - область интегрирования, $\widetilde{u}_{0}, \widetilde{u}_{n}$ - заданные числа, конечные разности при аппроксимации производных дают второй порядок точности [1-6]. Такой же порядок аппроксимации имеют методы численного интегрирования ряда краевых задач для уравнений в частных производных [4-10]. Второй порядок обусловлен тем, что при аппроксимации производных конечными разностями удерживается всего три члена разложения в ряд Тейлора искомого решения задачи.

Метод, использующий средства матричного исчисления и численного интегрирования разностных краевых задач для неоднородных ОДУ2, позволяющий увеличить количество удержанных членов до произвольного натурального числа в разложении искомой функции в ряд Тейлора, предложен в [11], при этом аппроксимация производных конечными разностями не использовалась. Оценка порядка аппроксимации предложенного в [11] матричного метода интегрирования для различных значений удержанного числа членов в рядах Тейлора дана в [12] для краевых задач с граничными условиями первого, второго и третьего рода.

Поставим целью исследовать вопросы устойчивости и, как следствие, сходимости матричного метода численного интегрирования разностных краевых задач для ОДУ2 с различными граничными условиями.

1. Обозначения и некоторые предшествующие результаты. Остановимся кратко на полученных в [11] результатах, придерживаясь следующих обозначений, принятых в [4]:

1) $D$ - область интегрирования, ограниченная отрезком $[a, b], D_{h}$ - узлы сетки, определяемые значениями $x_{i}=x_{0}+i h, i=1,2, \ldots, n, x_{0}=a$, $x_{n}=b, h=(b-a) / n, n+1-$ число узлов сетки;

2) $u(x)$ - непрерывная функция, являющаяся точным решением краевой задачи (1);

3) $[u]_{h}$ - сеточная функция, совпадающая с точным решением задачи (1) в узлах сетки $D_{h}$;

4) $u^{(h)}$ - искомая сеточная функция.

Для краткости примем для любой функции обозначение $\varphi\left(x_{i}\right)=\varphi_{i}$, где $x_{i}$ - узел сетки $D_{h}$.

В дальнейшем опустим индекс $h$ в наименованиях сеточных функций $[u]_{h}$, $u^{(h)}$ и будем оговаривать особо случаи, в которых будет использоваться непрерывная функция $u(x)$, являющаяся точным решением задачи $(1)$.

Для некоторого фиксированного $k \geqslant 2, k$-степень используемого многочлена Тейлора, в узлах сетки с номерами $i=1,2, \ldots, n-1$ составлена система уравнений, состоящая из $(k+1)$-го уравнения [11]. В систему внесены первые $k+1$ члена разложения в ряд Тейлора точной функции $u(x)$ в узлах $u_{i-1}, u_{i+1}$ соответственно и $k-1$ уравнение, которые получены дифференцированием 
по $x$ обеих частей уравнения $(1)$, так что

$$
\left(q_{i} u_{i}+p_{i} u_{i}^{\prime}+u_{i}^{\prime \prime}\right)^{(r)}=f_{i}^{(r)},
$$

где $r=0,1, \ldots, k-2$. Получено

$$
\left\{\begin{array}{l}
u_{i}-h u_{i}^{\prime}+\frac{h^{2}}{2 !} u_{i}^{\prime \prime}-\frac{h^{3}}{3 !} u_{i}^{\prime \prime \prime}+\cdots+(-1)^{k} \frac{h^{k}}{k !} u_{i}^{(k)}=u_{i-1}, \\
u_{i}+h u_{i}^{\prime}+\frac{h^{2}}{2 !} u_{i}^{\prime \prime}+\frac{h^{3}}{3 !} u_{i}^{\prime \prime \prime}+\cdots+\frac{h^{k}}{k !} u_{i}^{(k)}=u_{i+1}, \\
q_{i} u_{i}+p_{i} u_{i}^{\prime}+u_{i}^{\prime \prime}=f_{i}, \\
q_{i}^{\prime} u_{i}+\left(q_{i}+p_{i}^{\prime}\right) u_{i}^{\prime}+p_{i} u_{i}^{\prime \prime}+u_{i}^{\prime \prime \prime}=f_{i}^{\prime}, \\
\cdots \\
q_{i}^{(k-2)} u_{i}+\cdots+u_{i}^{(k)}=f_{i}^{(k-2)} .
\end{array}\right.
$$

В матричной форме система уравнений (2) имеет вид $A_{i}^{k} V_{i}^{k}=G_{i}^{k}$ в обозначениях

$$
\begin{gathered}
A_{i}^{k}=\left[\begin{array}{cccccc}
1 & -h & \frac{h^{2}}{2 !} & -\frac{h^{3}}{3 !} & \ldots & (-1)^{k} \frac{h^{k}}{k !} \\
1 & h & \frac{h^{2}}{2 !} & \frac{h^{3}}{3 !} & \ldots & \frac{h^{k}}{k !} \\
q_{i} & p_{i} & 1 & 0 & \ldots & 0 \\
q_{i}^{\prime} & q_{i}+p_{i}^{\prime} & p_{i} & 1 & \ldots & 0 \\
\ldots & \ldots & \ldots & \ldots & \ldots & \ldots \\
q_{i}^{(k-2)} & \ldots & \ldots & \ldots & \ldots & 1
\end{array}\right], \\
V_{i}^{k}=\left[\begin{array}{c}
u_{i} \\
u_{i}^{\prime} \\
u_{i}^{\prime \prime} \\
u_{i}^{\prime \prime \prime} \\
\ldots \\
u_{i}^{(k)}
\end{array}\right], \quad G_{i}^{k}=\left[\begin{array}{c}
u_{i-1} \\
u_{i+1} \\
f_{i} \\
f_{i}^{\prime} \\
\cdots \\
f_{i}^{(k-2)}
\end{array}\right]
\end{gathered}
$$

В дальнейшем верхний индекс будет означает степень многочлена Тейлора $k$, если речь не идёт о показателях алгебраических степеней, степенях производных, символов обратных матриц и транспонирования. При фиксированном номере $i$ узла сетки $D_{h}$ все элементы матрицы $A_{i}^{k}$ могут быть вычислены, следовательно, (2) является системой линейных алгебраических уравнений (СЛАУ). В предположении существования обратной матрицы $\left(A_{i}^{k}\right)^{-1}$ от матрицы $A_{i}^{k}$ найдено

$$
V_{i}^{k}=\left(A_{i}^{k}\right)^{-1} G_{i}^{k},
$$

или в координатной форме

$$
\begin{aligned}
& u_{i}=a_{11}^{k i} u_{i-1}+a_{12}^{k i} u_{i+1}+a_{13}^{k i} f_{i}+a_{14}^{k i} f_{i}^{\prime}+\cdots+a_{1, k+1}^{k i} f_{i}^{(k-2)}, \\
& u_{i}^{\prime}=a_{21}^{k i} u_{i-1}+a_{22}^{k i} u_{i+1}+a_{23}^{k i} f_{i}+a_{24}^{k i} f_{i}^{\prime}+\cdots+a_{2, k+1}^{k i} f_{i}^{(k-2)}, \\
& u_{i}^{\prime \prime}=a_{31}^{k i} u_{i-1}+a_{32}^{k i} u_{i+1}+a_{33}^{k i} f_{i}+a_{34}^{k i} f_{i}^{\prime}+\cdots+a_{3, k+1}^{k i} f_{i}^{(k-2)},
\end{aligned}
$$




$$
\begin{aligned}
u_{i}^{(k)}=a_{k+1,1}^{k i} u_{i-1}+a_{k+1,2}^{k i} & u_{i+1}+a_{k+1,3}^{k i} f_{i}+ \\
& +a_{k+1,4}^{k i} f_{i}^{\prime}+\cdots+a_{k+1, k+1}^{k i} f_{i}^{(k-2)},
\end{aligned}
$$

где $a_{l m}^{k i}$ - соответствующие элементы обратной матрицы $\left(A_{i}^{k}\right)^{-1}$ в узле с номером $i$. Из уравнений (4), каждое из которых при $i=1,2, \ldots, n-1$, есть разностное уравнение второго порядка, связывающее значения искомой сеточной функции $u_{i}$ в узлах $x_{i-1}, x_{i}, x_{i+1}$, при построении которого не использовались конечные разности для аппроксимации производных, составлена СЛАУ, решение которой для граничных условий $u_{0}=\widetilde{u}_{0}, u_{n}=\widetilde{u}_{n}$ даёт искомое приближённое решение $u_{i}, i=1,2, \ldots, n-1$, задачи $(1)$. В $[11,12]$ показано, что увеличение числа удержанных членов в разложении в ряд Тейлора функции $u(x)$, являющейся точным решением задачи $(1)$, приводит к уменьшению погрешности между точным и найденным приближённым решениями задачи в узлах сетки $D_{h}$. После нахождения решений $u_{i}, i=1,2, \ldots, n-1$, производные искомой сеточной функции вплоть до $k$-того порядка включительно могут быть найдены по формулам (5)-(7) во внутренних узлах сетки $D_{h}$. Значения производных в граничных узлах могут быть вычислены, например, в $x_{0}$, как

$$
u_{0}^{\prime}=u_{1}^{\prime}-h u_{1}^{\prime \prime}+\frac{h^{2}}{2 !} u_{1}^{\prime \prime \prime}+\cdots+(-1)^{k-1} \frac{h^{k-1}}{(k-1) !} u_{1}^{(k)}
$$

2. Некоторые предварительные оценки. В дальнейшем квадратные матрицы $A_{i}^{k}$ будем называть локальными матрицами, размерность которых равна $(k+1)$. Уравнение $(4)$ преобразуем во всех внутренних узлах сетки $D_{h}$ как

$$
-\frac{a_{11}^{k i}}{a_{13}^{k i}} u_{i-1}+\frac{u_{i}}{a_{13}^{k i}}-\frac{a_{12}^{k i}}{a_{13}^{k i}} u_{i+1}=f_{i}+\sum_{j=4}^{k+1} \frac{a_{1 j}^{k i}}{a_{13}^{k i}} f_{i}^{(j-3)},
$$

и, введя соответствующие обозначения в узле $x_{i}$ :

$$
\left\{\begin{array}{l}
a_{i}^{k}=-\frac{a_{11}^{k i}}{a_{13}^{k i}}=-\frac{M_{(11), i}^{k}}{M_{(13), i}^{k}}, \\
b_{i}^{k}=\frac{1}{a_{13}^{k i}}=\frac{\Delta_{i}^{k}}{M_{(13), i}^{k}}, \\
c_{i}^{k}=-\frac{a_{12}^{k i}}{a_{13}^{k i}}=-\frac{M_{(12), i}^{k}}{M_{(13), i}^{k}}, \\
d_{i j}^{k}=\frac{a_{1 j}^{k i}}{a_{13}^{k i}}=\frac{M_{(1 j), i}^{k}}{M_{(13), i}^{k}}, j=4,5, \ldots, k+1,
\end{array}\right.
$$

где $\Delta_{i}^{k}$ - определитель матрицы $A_{i}^{k} ; M_{(1 j), i}^{k}$ - алгебраическое дополнение элемента, расположенного на пересечении первой строки со столбцом с номером 
$j, j=1,2, \ldots, k+1$, матрицы $\left(A_{i}^{k}\right)^{\top}, \top$ - символ транспонирования, разностную краевую задачу, аппроксимирующую первую дифференциальную краевую задачу (1), запишем как

$$
\left\{\begin{array}{l}
a_{i}^{k} u_{i-1}+b_{i}^{k} u_{i}+c_{i}^{k} u_{i+1}=f_{i}+\sum_{j=4}^{k+1} d_{i j}^{k} f_{i}^{(j-3)}, i=1,2, \ldots, n-1, \\
u_{0}=\widetilde{u}_{0} \\
u_{n}=\widetilde{u}_{n}
\end{array}\right.
$$

или, по аналогии с [4], в компактной символьной форме

$$
L_{h}^{k} u=f_{h}^{k}
$$

причём наряду с введённым обозначением эту же задачу для краткости будем обозначать и как $L_{h}^{k}$. Дифференциальную краевую задачу, по аналогии с [4], будем обозначить как $L u=f$.

При $k=2$ из (8), (9) следует разностное уравнение

$$
\frac{2-h p_{i}}{2 h^{2}} u_{i-1}-\frac{2-h^{2} q_{i}}{h^{2}} u_{i}+\frac{2+h p_{i}}{2 h^{2}} u_{i+1}=f_{i}
$$

которое совпадает с уравнением традиционного метода численного интегрирования [4], использующего конечные разности при аппроксимации производных задачи (1).

Исходя их определения матрицы $A_{i}^{k}$ в форме (3) для любого натурального числа $k \geqslant 3$ имеем

$$
\begin{aligned}
& \Delta_{i}^{k}=\left|\begin{array}{cccccc}
1 & -h & \frac{h^{2}}{2 !} & \ldots & \frac{(-1)^{k-1} h^{k-1}}{(k-1) !} & \frac{(-1)^{k} h^{k}}{k !} \\
1 & h & \frac{h^{2}}{2 !} & \ldots & \frac{h^{k-1}}{(k-1) !} & \frac{h^{k}}{k !} \\
q_{i} & p_{i} & 1 & \ldots & 0 & 0 \\
\ldots & \ldots & \ldots & \ldots & \ldots & \ldots \\
q_{i}^{(k-3)} & r_{i} & s_{i} & \ldots & 1 & 0 \\
q_{i}^{(k-2)} & t_{i} & v_{i} & \ldots & w_{i} & 1
\end{array}\right|= \\
& \begin{array}{c}
=\frac{h^{k}}{k !}\left|\begin{array}{ccccc}
1 & h & \frac{h^{2}}{2 !} & \ldots & \frac{h^{k-1}}{(k-1) !} \\
q_{i} & p_{i} & 1 & \ldots & 0 \\
\ldots & \ldots & \ldots & \ldots & \ldots \\
q_{i}^{(k-3)} & r_{i} & s_{i} & \ldots & 1 \\
q_{i}^{(k-2)} & t_{i} & v_{i} & \ldots & w_{i}
\end{array}\right|+ \\
+\frac{(-1)^{k+1} h^{k}}{k !}\left|\begin{array}{ccccc}
1 & -h & \frac{h^{2}}{2 !} & \ldots & \frac{(-1)^{k-1} h^{k-1}}{(k-1) !} \\
q_{i} & p_{i} & 1 & \ldots & 0 \\
\ldots & \ldots & \ldots & \ldots & \ldots \\
q_{i}^{(k-3)} & r_{i} & s_{i} & \ldots & 1 \\
q_{i}^{(k-2)} & t_{i} & v_{i} & \ldots & w_{i}
\end{array}\right|+
\end{array}
\end{aligned}
$$




$$
\begin{aligned}
&++\left|\begin{array}{ccccc}
1 & -h & \frac{h^{2}}{2 !} & \ldots & \frac{(-1)^{k-1} h^{k-1}}{(k-1) !} \\
1 & h & \frac{h^{2}}{2 !} & \ldots & \frac{h^{k-1}}{(k-1) !} \\
q_{i} & p_{i} & 1 & \ldots & 0 \\
\ldots & \ldots & \ldots & \ldots & \ldots \\
q_{i}^{(k-3)} & r_{i} & s_{i} & \ldots & 1
\end{array}\right|= \\
&=\frac{h^{k}}{k !} \sum_{m=0}^{k-1} a_{m} h^{m}+\frac{(-1)^{k+1} h^{k}}{k !} \sum_{m=0}^{k-1} b_{m} h^{m}+\Delta_{i}^{k-1}=O\left(h^{k}\right)+\Delta_{i}^{k-1},
\end{aligned}
$$

где $r_{i}, s_{i}, t_{i}, v_{i}, w_{i}$ - некоторые записанные в узле $x_{i}$ функции от $p(x), q(x)$ и их производных; $a_{m}, b_{m}$ - независящие от $h$ величины; или

$$
\Delta_{i}^{k}=\Delta_{i}^{k-1}+O\left(h^{k}\right)
$$

Уменьшая степень используемого многочлена Тейлора в равенстве (12) на единицу, получим

$$
\Delta_{i}^{k-1}=\Delta_{i}^{k-2}+O\left(h^{k-1}\right) .
$$

Подставляя в (12) равенство (13), получим, пренебрегая старшими степенями, $\Delta_{i}^{k}=\Delta_{i}^{k-2}+O\left(h^{k-1}\right)$. Выполнение аналогичной процедуры ещё $k-4$ раза приводит к выражению

$$
\Delta_{i}^{k}=\Delta_{i}^{2}+O\left(h^{3}\right)
$$

в узле $x_{i}, i=1,2, \ldots, n-1$, сетки $D_{h}$.

Для первой краевой задачи (1) в [12] получены следующие оценки в узле с номером $i$ :

$$
M_{(1 j), i}^{k}=M_{(1 j), i}^{k-1}+O\left(h^{k}\right), j=1,2 .
$$

Выполняя описанную выше процедуру для равенства (15), найдём

$$
M_{(1 j), i}^{k}=M_{(1 j), i}^{2}+O\left(h^{3}\right), j=1,2 .
$$

В узлах сетки $D_{h}$ с номерами $i=2,3, \ldots, n-2$ второй и третьей краевых задач, граничные условия которых имеют, соответственно, вид

$$
u_{0}^{\prime}=\widetilde{u}_{0}, \quad u_{n}^{\prime}=\widetilde{u}_{n}
$$

и

$$
\alpha_{0} u_{0}+\beta_{0} u_{0}^{\prime}=\widetilde{z}_{0}, \quad \alpha_{1} u_{n}+\beta_{1} u_{n}^{\prime}=\widetilde{z}_{n},
$$

где $\widetilde{u}_{0}, \widetilde{u}_{n}, \alpha_{0}, \beta_{0}, \widetilde{z}_{0}, \alpha_{n}, \beta_{n}, \widetilde{z}_{n}$ - заданные числа, ситуация для оценок (14), (16) полностью совпадает с изложенной выше для первой краевой задачи. Найдём недостающие оценки $\Delta_{1}^{k}, M_{(11), 1}^{k}, M_{(12), 1}^{k}$ и $\Delta_{n-1}^{k}, M_{(11), n-1}^{k}, M_{(12), n-1}^{k}$ в узлах $x_{1}$ и $x_{n-1}$ для второй и третьей краевых задач $L_{h}^{k}$ соответственно.

Для третьей краевой задачи в узле $x_{1}$ при фиксированном $k$ запишем разложения

$$
u_{1}-h u_{1}^{\prime}+\frac{h^{2}}{2 !} u_{1}^{\prime \prime}-\frac{h^{3}}{3 !} u_{1}^{\prime \prime \prime}+\cdots+(-1)^{k} \frac{h^{k}}{k !} u_{1}^{(k)}=u_{0}
$$




$$
u_{1}^{\prime}-h u_{1}^{\prime \prime}+\frac{h^{2}}{2 !} u_{1}^{\prime \prime \prime}+\cdots+(-1)^{k-1} \frac{h^{k-1}}{(k-1) !} u_{1}^{(k)}=u_{0}^{\prime}
$$

Умножив обе части равенства (17) на $\alpha_{0}$, а $(18)$ - на $\beta_{0}$ и сложив, вместо первого уравнения системы $(2)$, записанного при $i=1$, получим

$$
\begin{array}{r}
\alpha_{0} u_{1}+\left(-\alpha_{0} h+\beta_{0}\right) u_{1}^{\prime}+\cdots+(-1)^{k-1}\left(-\alpha_{0} \frac{h^{k}}{k !}+\beta_{0} \frac{h^{k-1}}{(k-1) !}\right) u_{1}^{(k)}= \\
=\alpha_{0} u_{0}+\beta_{0} u_{0}^{\prime}=\widetilde{z}_{0} .
\end{array}
$$

Тогда локальная матрица $A_{1}^{k}$ третьей краевой задачи примет вид

$$
A_{1}^{k}=\left[\begin{array}{ccccc}
\alpha_{0} & -\alpha_{0} h+\beta_{0} & \alpha_{0} \frac{h^{2}}{2 !}-\beta_{0} h & \ldots & (-1)^{k-1}\left(-\alpha_{0} \frac{h^{k}}{k !}+\beta_{0} \frac{h^{k-1}}{(k-1) !}\right) \\
1 & h & \frac{h^{2}}{2 !} & \ldots & \frac{h^{k}}{k !} \\
q_{1} & p_{1} & 1 & \ldots & 0 \\
q_{1}^{\prime} & q_{1}+p_{1}^{\prime} & p_{1} & \ldots & 0 \\
\ldots & \ldots & \ldots & \ldots & \ldots \\
q_{1}^{(k-2)} & \ldots & \ldots & \ldots & 1
\end{array}\right] .
$$

Имеем

$$
\begin{aligned}
& \Delta_{1}^{k}=(-1)^{2 k+1}\left(-\alpha_{0} \frac{h^{k}}{k !}+\beta_{0} \frac{h^{k-1}}{(k-1) !}\right) \sum_{m=0}^{k-1} a_{m} h^{m}+ \\
& \quad+(-1)^{k+1} \frac{h^{k}}{k !} \sum_{m=0}^{k-1} b_{m} h^{m}+\Delta_{1}^{k-1}=O\left(h^{k-1}\right)+\Delta_{1}^{k-1} .
\end{aligned}
$$

Очевидно, полученное равенство (19) не нарушится при $\alpha_{0}=0$, что соответствует второй краевой задаче.

В работе [12] в узле $x_{1}$ показана справедливость оценки (16) при $j=1$ для второй и третьей краевых задач и справедливость оценки

$$
M_{(12), 1}^{k}=M_{(12), 1}^{k-1}+a_{k} h^{k-1}+b_{k} h^{k}
$$

где $a_{k}, b_{k}$ - не зависящие от $h$ величины для третьей краевой задачи, откуда непосредственно следует

$$
M_{(12), 1}^{k}=M_{(12), 1}^{k-1}+O\left(h^{k-1}\right)
$$

как для третьей, так и для второй краевой задачи. Из соотношений (19), (16) при $j=1$ и (20) имеем следующие оценки для второй и третьей краевых задач в узле $x_{1}$ :

$$
\begin{aligned}
\Delta_{1}^{k} & =\Delta_{1}^{2}+O\left(h^{2}\right), \\
M_{(11), 1}^{k} & =M_{(11), 1}^{2}+O\left(h^{3}\right), \\
M_{(12), 1}^{k} & =M_{(12), 1}^{2}+O\left(h^{2}\right) .
\end{aligned}
$$


Оценки $\Delta_{n-1}^{k}, M_{(11), n-1}^{k}, M_{(12), n-1}^{k}$ вычисляются аналогично изложенным выше способом.

3. Устойчивость матричного метода. Разностную краевую задачу

$$
a_{i} u_{i-1}+b_{i} u_{i}+c_{i} u_{i+1}=f_{i}, \quad u_{0}=\widetilde{u}_{0}, \quad u_{n}=\widetilde{u}_{n}, \quad i=1,2, \ldots, n-1,
$$

называют устойчивой [4], если она имеет единственное решение при произвольных $\widetilde{u}_{0}, \widetilde{u}_{n}, f_{1}, f_{2}, \ldots, f_{n-1}$ и имеет место оценка

$$
\|u\| \leqslant C\|f\|
$$

где $C$ - не зависящее от $h$ число, $\|w\|$ - некоторая норма вектора

$$
w=\left[\begin{array}{llll}
w_{0} & w_{1} & \ldots & w_{n}
\end{array}\right]^{\top},
$$

включая и выбранную в [4] норму

$$
\|w\|=\max \left(\left|w_{0}\right|,\left|w_{1}\right|, \ldots,\left|w_{n}\right|\right) .
$$

Правомерность выбора нормы в форме (26) для линейной разностной краевой задачи $L_{h}^{2}$ обоснована в [4].

При выборе нормы в форме (26) в неравенстве (25) следует положить $u=\left[\begin{array}{llll}u_{0} & u_{1} & \ldots & u_{n}\end{array}\right]^{\top}, f=\left[\begin{array}{lllll}\widetilde{u}_{0} & \widetilde{u}_{n} f_{1} f_{2} & \ldots & f_{n-1}\end{array}\right]^{\top}$.

Разностную краевую задачу (24) называют хорошо обусловленной [4], если она при всех достаточно больших $n$ имеет единственное решение при произвольных $\widetilde{u}_{0}, \widetilde{u}_{n}, f_{1}, f_{2}, \ldots, f_{n-1}$ и если значения $u_{0}, u_{1}, \ldots, u_{n}$, образующие решение, удовлетворяют неравенству

$$
\left|u_{i}\right| \leqslant P \max \left(\left|\widetilde{u}_{0}\right|,\left|\widetilde{u}_{n}\right|,\left|f_{1}\right|, \ldots,\left|f_{n-1}\right|\right), \quad i=0,1, \ldots, n,
$$

где $P$ - не зависящее от $h$ число. Выполнение неравенств (27) означает, что погрешности, допущенные при задании $\widetilde{u}_{0}, \widetilde{u}_{n}, f_{1}, f_{2}, \ldots, f_{n-1}$, не накапливаются и не приводят к возрастающим ошибкам с увеличением $n$ [4].

Неравенство (25) и совокупность неравенств (27) эквивалентны в смысле совпадения оценок ввиду выбора нормы в форме (26) и в силу совпадения их правых частей, следовательно, хорошая обусловленность разностной краевой задачи влечёт за собой её устойчивость.

Достаточным условием (критерием) хорошей обусловленности разностной краевой задачи (24) является выполнение неравенств

$$
\frac{\left|b_{i}\right|-\left|a_{i}+c_{i}\right|}{\left|b_{i}\right|+\left|a_{i}\right|+\left|c_{i}\right|} \geqslant \Theta>0, \quad i=1,2, \ldots, n-1,
$$

при условии гладкости коэффициентов

$$
\begin{cases}\left|a_{k}-a_{m}\right| \leqslant D\left|\frac{k-m}{n}\right|^{r}, & \left|b_{k}-b_{m}\right| \leqslant D\left|\frac{k-m}{n}\right|^{r} \\ \left|c_{k}-c_{m}\right| \leqslant D\left|\frac{k-m}{n}\right|^{r}, & D>0, r>0\end{cases}
$$

в случае их ограниченности

$$
\left|a_{i}\right| \leqslant Q, \quad\left|b_{i}\right| \leqslant Q, \quad\left|c_{i}\right| \leqslant Q, \quad i=1,2, \ldots, n-1
$$


и в предположении немалости одновременно

$$
g_{i}=\max \left(\left|a_{i}\right|,\left|b_{i}\right|,\left|c_{i}\right|\right) \geqslant B>0, i=1,2, \ldots, n-1
$$

где числа $\Theta, Q, B$ не зависят от номера узла $i$ и значения $n$ [4].

Для первой разностной краевой задачи $L_{h}^{k}, k \geqslant 3$, с учётом $(9),(14)$ имеем

$$
\begin{aligned}
& \frac{\left|b_{i}^{k}\right|-\left|a_{i}^{k}+c_{i}^{k}\right|}{\left|b_{i}^{k}\right|+\left|a_{i}^{k}\right|+\left|c_{i}^{k}\right|}= \\
& =\frac{\left|\frac{\Delta_{i}^{k}}{M_{(13), i}^{k}}\right|-\left|-\frac{M_{(11), i}^{k}}{M_{(13), i}^{k}}-\frac{M_{(12), i}^{k}}{M_{(13), i}^{k}}\right|}{\left|\frac{M_{(1), i}^{k}}{M_{(13), i}^{k}}\right|+\left|\frac{\Delta_{i}^{k}}{M_{(13), i}^{k}}\right|+\left|\frac{M_{(12), i}^{k}}{M_{(13), i}^{k}}\right|}=\frac{\left|\Delta_{i}^{k}\right|-\left|M_{(11), i}^{k}+M_{(12), i}^{k}\right|}{\left|M_{(11), i}^{k}\right|+\left|\Delta_{i}^{k}\right|+\left|M_{(12), i}^{k}\right|}= \\
& =\frac{\left|\Delta_{i}^{2}+O\left(h^{3}\right)\right|-\left|M_{(11), i}^{2}+M_{(12), i}^{2}+O\left(h^{3}\right)\right|}{\left|M_{(11), i}^{2}+O\left(h^{3}\right)\right|+\left|\Delta_{i}^{2}+O\left(h^{3}\right)\right|+\left|M_{(12), i}^{2}+O\left(h^{3}\right)\right|} \approx \\
& \approx \frac{\left|\Delta_{i}^{2}\right|-\left|M_{(11), i}^{2}+M_{(12), i}^{2}\right|}{\left|M_{(11), i}^{2}\right|+\left|\Delta_{i}^{2}\right|+\left|M_{(12), i}^{2}\right|}=\frac{\left|\frac{\Delta_{i}^{2}}{M_{(13), i}^{2}}\right|-\left|-\frac{M_{(11), i}^{2}}{M_{(13), i}^{k}}-\frac{M_{(12), i}^{2}}{M_{(13), i}^{2}}\right|}{\left|\frac{M_{(11), i}^{2}}{M_{(13), i}^{2}}\right|+\left|\frac{\Delta_{i}^{2}}{M_{(13), i}^{2}}\right|+\left|\frac{M_{(12), i}^{2}}{M_{(13), i}^{k}}\right|}= \\
& =\frac{\left|b_{i}^{2}\right|-\left|a_{i}^{2}+c_{i}^{2}\right|}{\left|b_{i}^{2}\right|+\left|a_{i}^{2}\right|+\left|c_{i}^{2}\right|},
\end{aligned}
$$

откуда следует, что если устойчивой является краевая задача $L_{h}^{2}$, т.е. наряду с выполнением (29)-(31) имеет место неравенство

$$
\frac{\left|b_{i}^{2}\right|-\left|a_{i}^{2}+c_{i}^{2}\right|}{\left|b_{i}^{2}\right|+\left|a_{i}^{2}\right|+\left|c_{i}^{2}\right|} \geqslant \Theta>0, \quad i=1,2, \ldots, n-1,
$$

то устойчивой является и задача $L_{h}^{k}$ при условии справедливости оценок $(29)-$ (31) для неё.

Выявим достаточное условие устойчивости для задачи $L_{h}^{2}$. В силу того, что, как видно из (11),

$$
a_{i}^{2}+c_{i}^{2}=\frac{1}{h^{2}}-\frac{p_{i}}{2 h}+\frac{1}{h^{2}}+\frac{p_{i}}{2 h}=\frac{2}{h^{2}}>0,
$$

имеем

$$
\left|b_{i}^{2}\right|=\left|\frac{\Delta_{i}^{2}}{M_{(13), i}^{2}}\right|=\left|\frac{2-h^{2} q_{i}}{h^{2}}\right|=\left|\frac{2}{h^{2}}-q_{i}\right|=\left|a_{i}^{2}+c_{i}^{2}-q_{i}\right| .
$$

Тогда, по крайней мере при

$$
q(x)<0, \quad x \in[a, b],
$$


из равенства (34) следует $\left|b_{i}^{2}\right|=\left|a_{i}^{2}+c_{i}^{2}\right|+\left|q_{i}\right|$ или

$$
\left|b_{i}^{2}\right|-\left|a_{i}^{2}+c_{i}^{2}\right|=\left|q_{i}\right|, \quad i=1,2, \ldots, n-1 .
$$

При условии справедливости оценок (29)-(31) для первых разностных краевых задач $L_{h}^{k}, k \geqslant 2$, из (33) с учётом (36) имеем оценку

$$
\frac{\left|b_{i}^{2}\right|-\left|a_{i}^{2}+c_{i}^{2}\right|}{\left|b_{i}^{2}\right|+\left|a_{i}^{2}\right|+\left|c_{i}^{2}\right|}=\frac{\left|q_{i}\right|}{\left|b_{i}^{2}\right|+\left|a_{i}^{2}\right|+\left|c_{i}^{2}\right|} \geqslant \Theta>0, \quad i=1,2, \ldots, n-1,
$$

где

$$
\Theta=\frac{1}{3 B} \min _{[a, b]}|q(x)|
$$

что обеспечивает устойчивость задачи $L_{h}^{2}$ и, как следствие, задачи $L_{h}^{k}$ при выполнении условия (35).

Для второй и третьей разностных краевых задач $L_{h}^{k}, k \geqslant 2$, для узлов сетки $x_{i}, i=2,3, \ldots, n-2$, ситуация полностью совпадает с изложенной выше. Для узлов $x_{1}, x_{n}$ подстановка в левую часть (32) оценок (21)-(23) вместо (14), (16) приводит к аналогичному (32) соотношению. Следовательно, методы оценки устойчивости, разработанные в классическом методе численного интегрирования разностной краевой задачи $L_{h}^{2}[4,6,8]$, могут быть использованы при исследовании матричным методом задачи $L_{h}^{k}, k \geqslant 3$.

В [4] доказана эквивалентность достаточного критерия устойчивости (28)(31) задачи (24) следующему условию:

$$
\left|b_{i}\right| \geqslant\left|a_{i}\right|+\left|c_{i}\right|+\delta, \quad i=1,2, \ldots, n-1,
$$

где $\delta>0$ не зависит от номера узла $i$ и числа $n$ [4].

При $i=1, i=n-1$ разностное уравнение (24) содержит только две неизвестные: $u_{1}, u_{2}$ при $i=1$ и $u_{n-2}, u_{n-1}$ при $i=n-1$; вследствие чего критерий устойчивости в [5] представлен как

$$
\begin{gathered}
\left|b_{1}\right| \geqslant\left|c_{1}\right| \\
\left|b_{i}\right| \geqslant\left|a_{i}\right|+\left|c_{i}\right|, \quad i=2,3, \ldots, n-2, \\
\left|b_{n-1}\right| \geqslant\left|a_{n-1}\right|
\end{gathered}
$$

причём строгое неравенство должно иметь место хотя бы в одном из выражений (37)-(39).

4. Сходимость матричного метода. Решение $u_{0}, u_{1}, \ldots, u_{n}$ разностной краевой задачи $L_{h}^{k} u=f_{h}^{k}$ при измельчении сетки $D_{h}$ сходится к решению дифференциальной краевой задачи $L u=f$, если

$$
\|[u]-u\| \rightarrow 0 \quad \text { при } \quad h \rightarrow 0 .
$$

Если при этом имеет место неравенство

$$
\|[u]-u\| \leqslant P h^{k}
$$


где $P, k$ - не зависящие от $h$ числа, то в этом случае говорят, что разностная краевая задача имеет $k$-тый порядок точности или что имеет место сходимость порядка $h^{k}$ (задача сходится со скоростью $\left.O\left(h^{k}\right)\right)[4,6]$.

Разностная краевая задача $L_{h}^{k} u=f_{h}^{k}$ аппроксимирует дифференциальную краевую задачу $L u=f$, если

$$
\left\|\delta f_{h}^{k}\right\| \rightarrow 0 \quad \text { при } \quad h \rightarrow 0,
$$

где $\delta f_{i h}^{k}$ - невязка в узле с номером $i$ сетки $D_{h}$, характеризующая отличия, возникающие при подстановке сеточной функции $\left[u_{i}\right]$ в разностную краевую задачу; иными словами, подстановка $[u]$ в $L_{h}^{k} u=f_{h}^{k}$ приводит к выражению

$$
L_{h}^{k}[u]=f_{h}^{k}+\delta f_{h}^{k}
$$

Если при этом выполнено неравенство

$$
\left\|\delta f_{h}^{k}\right\| \leqslant C h^{k}
$$

где $C, k$ - не зависящие от $h$ числа, то говорят, что имеет место аппроксимация порядка $h^{k}$, или порядка $k$ относительно величины $h[4,6]$.

Согласно доказанной в [4] теореме, если разностная краевая задача $L_{h}^{k} u=$ $=f_{h}^{k}$ аппроксимирует дифференциальную краевую задачу $L u=f$ с порядком $h^{k}$ и является устойчивой, то решение разностной краевой задачи $u_{i}$ сходится к $\left[u_{i}\right], i=1,2, \ldots, n-1$, причём порядок точности совпадает с порядком аппроксимации $k$ относительно величины $h$. Аналогичный вывод сделан и в работе [6].

Для первой разностной краевой задачи (10) в работе [12] получены следующие оценки:

$$
\begin{gathered}
\left\|\delta f_{h}^{k}\right\| \leqslant C h^{k} \quad \text { для чётного } k ; \\
\left\|\delta f_{h}^{k}\right\| \leqslant C h^{k-1} \quad \text { для нечётного } k .
\end{gathered}
$$

Откуда имеем, что задачи $L_{h}^{2 m}$ и $L_{h}^{2 m+1}$ для любого натурального числа $m \geqslant 1$ имеют одинаковый порядок аппроксимации. Следовательно, при условии выполнения критерия устойчивости получаем, что первая разностная краевая задача сходится со скоростью $O\left(h^{k}\right)$ для чётного $k$ и со скоростью $O\left(h^{k-1}\right)$ для нечётного $k$.

В работе [12] показано, что порядок аппроксимации второй и третьей краевых задач $L_{h}^{k} u=f_{h}^{k}$ на единицу меньше коэффициента $k$ независимо от его чётности, следовательно, в этом случае задача $L_{h}^{k}$ сходится со скоростью $O\left(h^{k-1}\right)$. Использование предложенного в [12] метода повышения порядка аппроксимации на единицу приведёт к скорости сходимости $O\left(h^{k}\right)$ второй и третьей краевых задач $L_{h}^{k}$.

5. Численный эксперимент. При выполнении численного эксперимента для разностной задачи $L_{h}^{k}$ в качестве меры суммарной оценки относительной погрешности в узлах сетки $D_{h}$ принята величина

$$
E^{k}=\frac{\sqrt{\sum_{i=1}^{n-1}\left(\left[u_{i}\right]-u_{i}\right)^{2}}}{\sum_{i=1}^{n-1}\left|\left[u_{i}\right]\right|} \cdot 100 \%,
$$


которую можно трактовать как некий аналог коэффициента вариации в статистике, являющегося безразмерной мерой рассеяния (разброса) и характеризующего эту меру в процентах [13]. При выполнении численного эксперимента полагалось, что $n=20$.

Рассмотрим ОДУ2

$$
u^{\prime \prime}-\frac{2}{x} u^{\prime}-\frac{4}{x^{2}} u=\frac{\sin x}{x}+\frac{4 \cos x}{x^{2}},
$$

имеющее аналитическое решение

$$
u=C_{1} x^{4}+\frac{C_{2}-\sin x}{x}
$$

для которого при любых граничных условиях выполнено обеспечивающее устойчивость условие (35).

В табл. 1-3 приведены результаты исследования краевых задач для ОДУ2 (40) с граничными условиями первого рода

$$
u(1)=-1.827, \quad u(5)=8.742,
$$

с граничными условиями третьего рода

$$
2 u(1)-u^{\prime}(1)=-5.012, \quad u(5)+2 u^{\prime}(5)=-22.631
$$

и с граничными условиями смешанного типа

$$
2 u(1)-u^{\prime}(1)=-5.012, \quad u^{\prime}(5)=6.945 .
$$

В третьей строке табл. 1 (и ниже в табл. 4) указано число узлов $n$ сетки $D_{h}$, при котором была достигнута погрешность $E^{9}$, соответствующая задаче $L_{h}^{9}$, а в четвёртой строке для числа узлов $n$ сетки $D_{h}$ указано суммарное число арифметических операций $Q$ при вычислении обратных матриц от локальных матриц $A_{i}^{k}, i=1,2, \ldots, n-1$, когда каждое из них вычислялось по формуле [14]:

$$
Q=\frac{8}{3}(k+1)^{3}-\frac{1}{2}(k+1)^{2}-\frac{1}{6}(k+1)+1 .
$$

Отметим, что в задачах для уравнения (40) при всех $k=2,3, \ldots, 9$ в узлах $x_{i}, i=2,3, \ldots, n-2$, сетки $D_{h}$ с граничными условиями первого рода (41), условиями третьего рода (42) и условиями смешанного типа (43) устойчивость, а вместе с ней и сходимость, обеспечивались за счёт выполнения неравенства (35). В узле $x_{1}$ устойчивость обеспечена тем, что

$$
\frac{\left|c_{1}\right|}{\left|b_{1}\right|} \leqslant 6.089 \cdot 10^{-1}
$$

для всех упомянутых выше граничных условий, что повлекло за собой выполнение (37). В узле $x_{n-1}$ выполнение условия

$$
\frac{\left|a_{n-1}\right|}{\left|b_{n-1}\right|} \leqslant 9.176 \cdot 10^{-1}
$$


повлекло за собой выполнение обеспечивающего устойчивость неравенства (39).

Аналогичная ситуация наблюдалась при исследовании краевых задач для ряда других ОДУ2, в частности для

$$
u^{\prime \prime}+\frac{1}{x} u^{\prime}-\frac{1}{x^{2}} u=1,
$$

для которого условия устойчивости (35), (37), (39) оказались не нарушенными.

Рассмотрим ОДУ2

$$
u^{\prime \prime}-\frac{2}{x} u^{\prime}+\frac{2}{x^{2}} u=x \cos x,
$$

имеющее аналитическое решение $u=C_{1} x+C_{1} x^{2}-x \cos x$, для которого при любых граничных условиях нарушено обеспечивающее устойчивость условие $(35)$.

В табл. 4-6 приведены результаты исследования краевых задач для ОДУ2 (44) с граничными условиями первого рода

$$
u(5)=22.331, \quad u(13)=23.953
$$

и с граничными условиями смешанного типа:

$$
\begin{array}{ll}
u(5)+3 u^{\prime}(5)=17.597, & u(13)=23.953 \\
u(5)+3 u^{\prime}(5)=17.597, & u^{\prime}(13)=4.054 .
\end{array}
$$

Приведённые в табл. 4-6 результаты свидетельствуют о наличии сходимости краевых задач для уравнения (44) с граничными условиями (45)-(47) для всех $k=2,3, \ldots, 9$, однако неравенство (35), которое обеспечивает выполнение достаточного условия устойчивости в узлах сетки $x_{i}, i=2,3, \ldots, n-2$, было нарушено для всех типов используемых граничных условий. Неравенства (37), (39) были нарушены для задач с граничными условиями смешанного типа, в частности

$$
\frac{\left|c_{1}\right|}{\left|b_{1}\right|} \geqslant 1.278
$$

для условий (46), (47) и

$$
\frac{\left|a_{n-1}\right|}{\left|b_{n-1}\right|} \geqslant 1.003
$$

для граничного условия (47). Полученные результаты объясняются лишь достаточностью условий устойчивости (35), (37), (39).

Аналогичная ситуация наблюдалась при исследовании краевых задач для ряда других ОДУ2, в частности для

$$
u^{\prime \prime}+\frac{2}{x} u^{\prime}+u=1 .
$$

Очевидно, для ОДУ2

$$
u^{\prime \prime}-\frac{2}{x} u^{\prime}-\frac{4}{x^{2}} u=x \ln x
$$




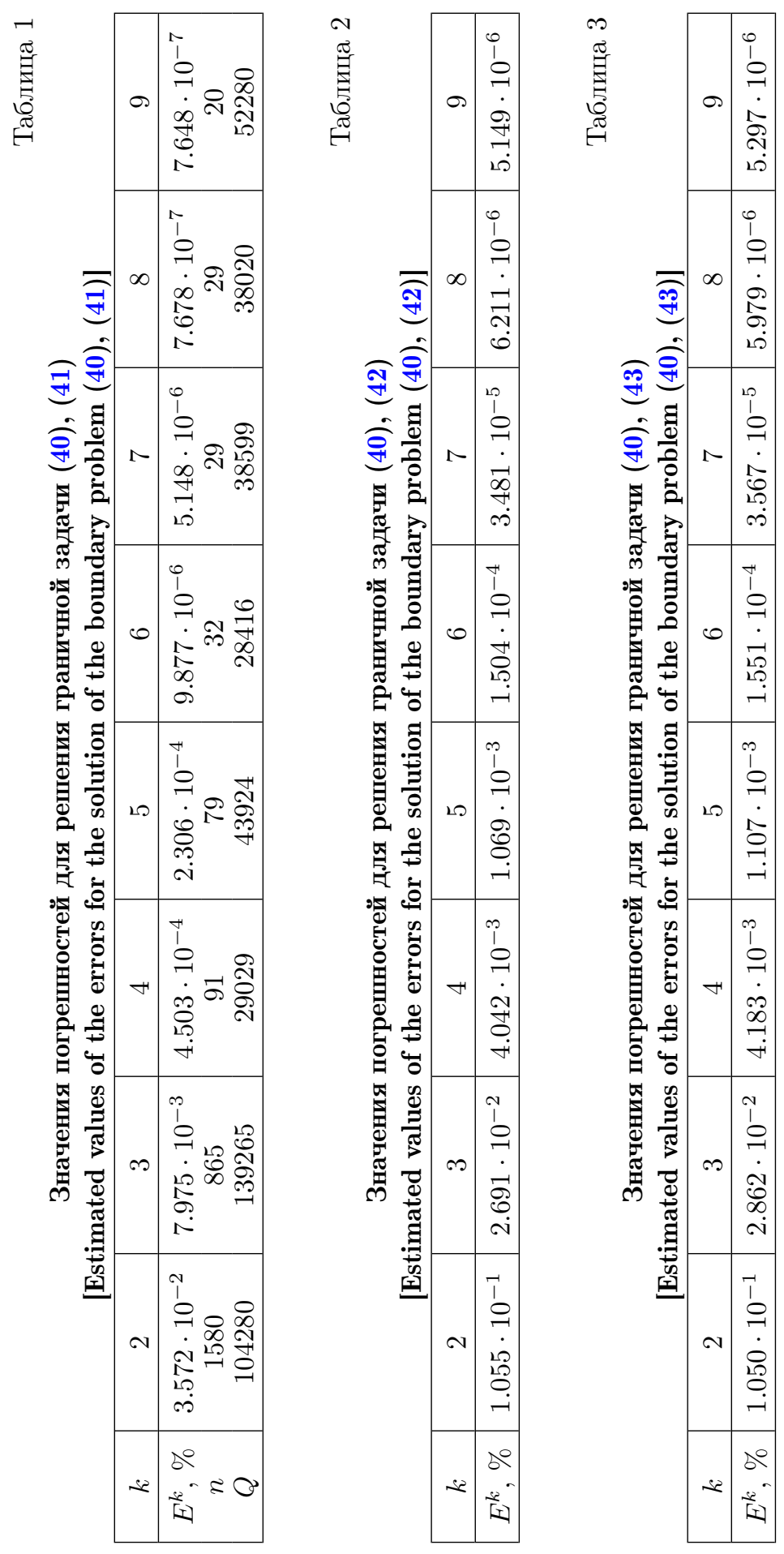




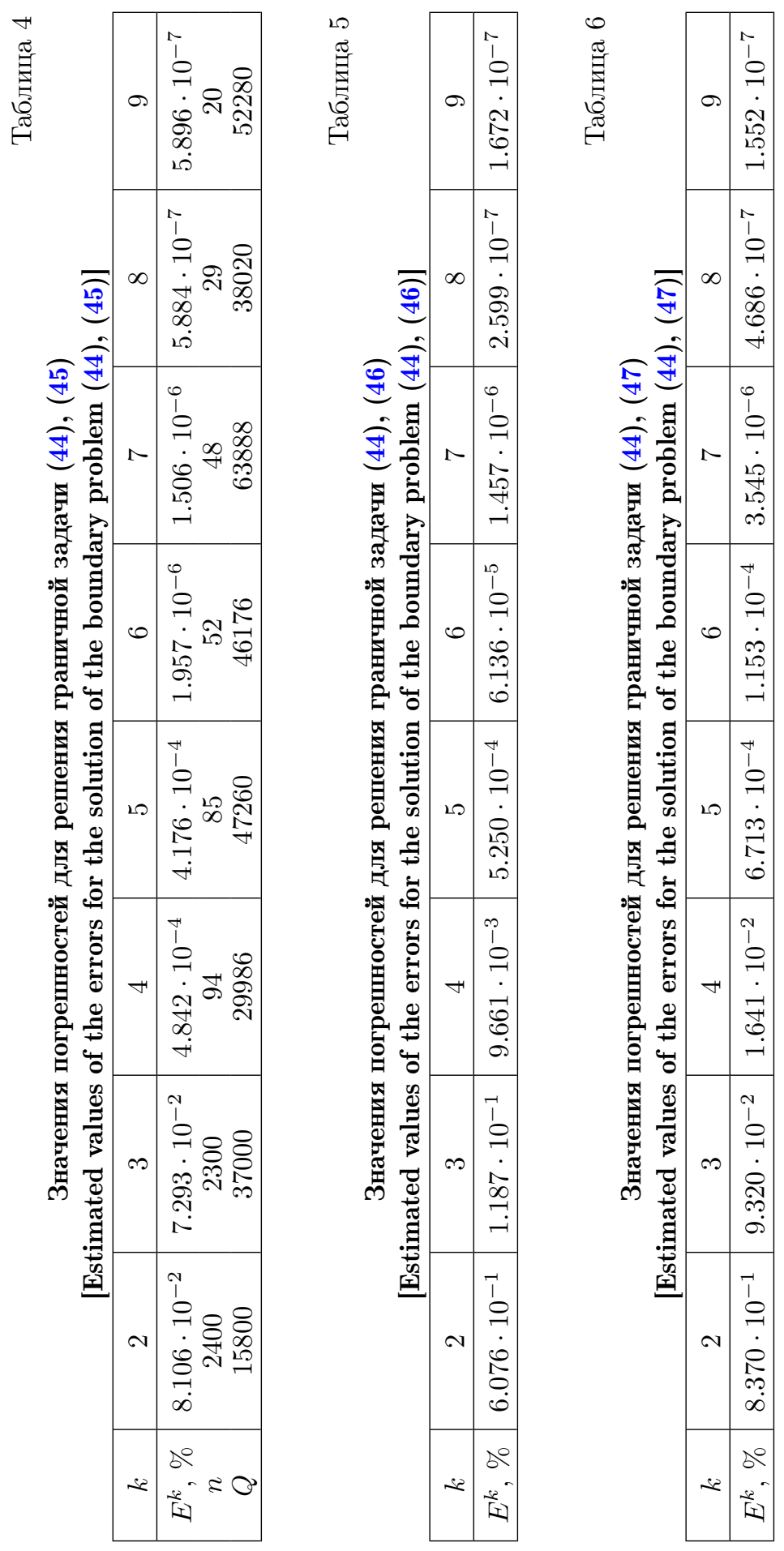


условие (35) выполнено для всех $k=2,3, \ldots, 9$ в узлах $x_{i}, i=2,3, \ldots, n-2$ любого отрезка интегрирования $[a, b]$. Численный эксперимент для ОДУ2 (48) со смешанными граничными условиями

$$
u(-5)+u^{\prime}(-5)=-33.617, \quad u(-1)=-1.050
$$

показал отсутствие сходимости для всех $k=2,3, \ldots, 9$. Оказалось, что

$$
\frac{\left|c_{1}\right|}{\left|b_{1}\right|} \geqslant 1.237,
$$

причём с ростом $k$ погрешность увеличивается от $E^{2}=1215.2$ до $E^{9}=$ $=70796.6$. Для того же ОДУ2 со смешанными граничными условиями

$$
u(1)+u^{\prime}(1)=-2.594, \quad u(5)=151.979
$$

оказалось, что

$$
\frac{\left|c_{1}\right|}{\left|b_{1}\right|} \leqslant 0.802,
$$

а сама разностная краевая задача оказалась сходящейся, чего и следовало ожидать.

Результаты численного эксперимента свидетельствуют, что выполнение достаточных условий устойчивости (35), (37), (39) обеспечивает устойчивость и, как следствие, сходимость разностных краевых задач для ОДУ2. При невыполнении хотя бы одного из условий $(35),(37),(39)$ вопрос о сходимости остаётся открытым.

Анализ четвёртых строк в табл. 1, 4 ещё раз подтверждает сделанный в [12] вывод о целесообразности использования на практике задач $L_{h}^{k}$ с чётным номером $k$.

Выводы. В настоящей работе теоретически установлены следующие факты.

1. Достаточный критерий устойчивости матричного метода при использовании многочленов Тейлора степени три и выше эквивалентен известному достаточному критерию устойчивости традиционного метода сеток для численного интегрирования разностных краевых задач для ОДУ2 с граничными условиями первого, второго и третьего рода.

2. Скорость сходимости матричного метода интегрирования разностных краевых задач с граничными условиями первого рода пропорциональна степени используемого многочлена Тейлора в случае, когда эта степень является чётной, и пропорциональна числу, меньшему степени на единицу, когда эта степень является нечётной.

3. При интегрировании разностных краевых задач с граничными условиями второго и третьего рода скорость сходимости метода пропорциональна степени используемого многочлена Тейлора независимо от его чётности и меньше него на единицу.

\section{ORCID}

Владимир Николаевич Маклаков: http://orcid.org/0000-0003-1644-7424 


\section{БИБЛИОГРАФИЧЕСКИЙ СПИСОК}

1. Keller H. B. Accurate difference methods for nonlinear two-point boundary value problems // SIAM J. Numer. Anal., 1974. vol.11, no. 2. pp. 305-320. doi: 10.1137/0711028.

2. Lentini M., Pereyra V. A variable order finite difference method for nonlinear multipoint boundary value problems // Math. Comp., 1974. vol.28, no.128. pp. 981-1003. doi: 10 . 1090/s0025-5718-1974-0386281-4.

3. Keller H. B. Numerical solution of boundary value problems for ordinary differential equations: Survey and some resent results on difference methods / Numerical solutions of boundary value problems for ordinary differential equations; ed. A. K. Aziz. New York: Academic Press, 1975. pp. 27-88. doi: 10.1016/b978-0-12-068660-5.50007-7.

4. Годунов С. К., Рябенький В. С. Разностные схемы. М.: Наука, 1977. 439 с.

5. Формалеев В. Ф., Ревизников Д. Л. Численные методы. М.: Физматлит, 2004. 400 с.

6. Самарский А. А. Теория разностных схем. М.: Наука, 1977. 656 с.

7. Самарский А. А., Гулин А. В. Численные методы. М.: Наука, 1989. 432 с.

8. Самарский А. А., Гулин А. В. Устойчивость разностных схем. М.: Наука, 1973. 416 с.

9. Boutayeb A., Chetouani A. Global extrapolations of numerical methods for a parabolic problem with nonlocal boundary conditions// International Journal of Computer Mathematics, 2003. vol.80, no.6. pp. 789-797. doi : 10.1080/0020716021000039209.

10. Boutayeb A., Chetouani A. A numerical comparison of different methods applied to the solution of problems with non local boundary conditions // Applied Mathematical Sciences, 2007. vol. 1, no. 44. pp. 2173-2185, http://www.m-hikari.com/ams/ams-password-2007/ ams-password41-44-2007/boutayebAMS41-44-2007.pdf.

11. Радченко В. П., Усов А. А. Модификация сеточных методов решения линейных дифференциальных уравнений с переменными коэффициентами на основе тейлоровских разложений // Вестн. Сам. гос. техн. ун-та. Сер. Физ.-мат. науки, 2008. №2(17). C. 60-65. doi: 10.14498/vsgtu646.

12. Маклаков В. Н. Оценка порядка аппроксимации матричного метода численного интегрирования краевых задач для линейных неоднородных обыкновенных дифференциальных уравнений второго порядка // Вестн. Сам. гос. техн. ун-та. Сер. Физ.мат. науки, 2014. №3(36). С. 143-160. doi: 10.14498/vsgtu1364; Опечатка// Вестн. Сам. гос. техн. ун-та. Сер. Физ.-мат. науки, 2014. №4(37). С. 187. doi:10.14498/ vsgtu1409.

13. Закс Л. Статистическое оценивание. М.: Статистика, 1976. 598 с.

14. Турчак Л. И. Основы численных методов. М.: Наука, 1987. 320 с.

Поступила в редакцию 06/IV/2015;

в окончательном варианте $-17 / \mathrm{V} / 2015$;

принята в печать - 08/VIII/2015. 
Vestn. Samar. Gos. Techn. Un-ta. Ser. Fiz.-mat. nauki

[J. Samara State Tech. Univ., Ser. Phys. \& Math. Sci.], 2015, vol. 19, no. 3, pp. 559-577

ISSN: 2310-7081 (online), 1991-8615 (print)

doi: http://dx.doi.org/10.14498/vsgtu1426

MSC: 34B99

\title{
CONVERGENCE OF THE MATRIX METHOD \\ OF NUMERICAL INTEGRATION OF THE BOUNDARY VALUE PROBLEMS FOR LINEAR NONHOMOGENEOUS ORDINARY DIFFERENTIAL SECOND ORDER EQUATIONS WITH VARIABLE COEFFICIENTS
}

\author{
V. N. Maklakov
}

Samara State Technical University,

244, Molodogvardeyskaya st., Samara, 443100, Russian Federation.

\begin{abstract}
The problems of stability and convergence of previously proposed matrix method of numerical integration of boundary value problems with boundary conditions of the first, second and third kinds of nonhomogeneous linear ordinary differential second order equations with variable coefficients are considered. Using of the Taylor polynomials of arbitrary degrees allowed to increase the approximation order of the method to an arbitrary natural number and to refuse from the approximation of derivatives by finite differences. When choosing the second degree Taylor polynomials the equation of the method coincided with the known equations of the traditional method of numerical integration of the boundary value problems where the derivatives are approximated by finite differences. It was shown that a sufficient criterion of stability when used in the method of Taylor polynomials of the third degree and more coincides with the sufficient criterion of stability of the traditional grid method for the numerical integration of boundary value problems with boundary conditions of the first, second and third kind. Theoretically, it is established that the degree of convergence of the matrix method for integration of boundary value problems with boundary conditions of the first kind is proportional to the degree of the used Taylor polynomials in the case, when the degree is even, and is proportional to the number that is one less than the degree if it is odd; when integrating the boundary value problems with boundary conditions of the second and third kind the degree of convergence of the method is proportional to the degree of the used Taylor polynomials regardless of its parity and one less than it. The obtained theoretical results are confirmed by numerical experiments.
\end{abstract}

Keywords: ordinary differential equations, boundary value problems, approximation order, stability, convergence, numerical methods, Taylor polynomials.

(C) 2015 Samara State Technical University.

Please cite this article in press as:

Maklakov V. N. Convergence of the matrix method of numerical integration of the boundary value problems for linear nonhomogeneous ordinary differential second order equations with variable coefficients, Vestn. Samar. Gos. Tekhn. Univ., Ser. Fiz.-Mat. Nauki [J. Samara State Tech. Univ., Ser. Phys. \& Math. Sci.], 2015, vol. 19, no. 3, pp. 559-577. doi: 10.14498/vsgtu1426. (In Russian)

\section{Author Details:}

Vladimir N. Maklakov (Cand. Phys. \& Math. Sci.), Associate Professor, Dept. of Higher Mathematics and Applied Informatics. 
doi: http://dx.doi.org/10.14498/vsgtu1426

\section{ORCID}

Vladimir N. Maklakov: http://orcid.org/0000-0003-1644-7424

\section{REFERENCES}

1. Keller H. B. Accurate difference methods for nonlinear two-point boundary value problems, SIAM J. Numer. Anal., 1974, vol. 11, no. 2, pp. 305-320. doi : 10.1137/0711028.

2. Lentini M., Pereyra V. A variable order finite difference method for nonlinear multipoint boundary value problems, Math. Comp., 1974, vol. 28, no. 128, pp. 981-1003. doi: 10.1090/ s0025-5718-1974-0386281-4.

3. Keller H. B. Numerical solution of boundary value problems for ordinary differential equations: Survey and some resent results on difference methods, Numerical solutions of boundary value problems for ordinary differential equations; ed. A. K. Aziz. New York, Academic Press, 1975, pp. 27-88. doi: 10.1016/b978-0-12-068660-5.50007-7.

4. Godunov S. K., Ryaben'kij V. S. Difference schemes. An introduction to the underlying theory, Studies in Mathematics and its Applications, vol. 19. Amsterdam, North-Holland, 1987, xvii+489 pp.

5. Formaleev V. F., Reviznikov D. L. Chislennye metody [Numerical Methods]. Moscow, Fizmatlit, 2004, 400 pp. (In Russian)

6. Samarskii A. The Theory of Difference Schemes, Pure and Applied Mathematics, vol. 240. New York, Bassel, Marcel Dekker, 2001, 786 pp. doi: 10.1201/9780203908518.

7. Samarskii A. A., Gulin A. V. Chislennye metody [Numerical methods]. Moscow, Nauka, 1989, 432 pp. (In Russian)

8. Samarskii A. A., Gulin A. V. Ustoichivost' raznostnykh skhem [Stability Theory of Difference Schemes]. Moscow, Nauka, 1973, 416 pp. (In Russian)

9. Boutayeb A., Chetouani A. Global extrapolations of numerical methods for a parabolic problem with nonlocal boundary conditions, International Journal of Computer Mathematics, 2003, vol. 80, no. 6, pp. 789-797. doi : 10.1080/0020716021000039209.

10. Boutayeb A., Chetouani A. A numerical comparison of different methods applied to the solution of problems with non local boundary conditions, Applied Mathematical Sciences, 2007, vol.1, no.44, pp. 2173-2185, http://www.m-hikari.com/ams/ams-password-2007/ ams-password41-44-2007/boutayebAMS41-44-2007.pdf.

11. Radchenko V. P., Usov A. A. Modified grid method for solving linear differential equation equipped with variable coefficients based on Taylor series, Vestn. Samar. Gos. Tekhn. Univ. Ser. Fiz.-Mat. Nauki [J. Samara State Tech. Univ., Ser. Phys. \& Math. Sci.], 2008, no. 2(17), pp. 60-65 (In Russian). doi: 10.14498/vsgtu646.

12. Maklakov V. N. Estimation of the Order of the Matrix Method Approximation of Numerical Integration of Boundary-Value Problems for Inhomogeneous Linear Ordinary Differential Equations of the Second Order, Vestn. Samar. Gos. Tekhn. Univ. Ser. Fiz.-Mat. Nauki [J. Samara State Tech. Univ., Ser. Phys. \& Math. Sci.], 2014, no. 3(36), pp. 143-160 (In Russian). doi: 10.14498/vsgtu1364 ; Erratum, Vestn. Samar. Gos. Tekhn. Univ. Ser. Fiz.-Mat. Nauki [J. Samara State Tech. Univ., Ser. Phys. \& Math. Sci.], 2014, no. 4(37), pp. 187. doi : 10.14498/vsgtu1409.

13. Zaks L. Statisticheskoe otsenivanie [Statistical estimation]. Moscow, Statistika, 1976, 598 pp. (In Russian)

14. Turchak L. I. Osnovy chislennykh metodov [The fundamentals of numerical methods]. Moscow, Nauka, 1987, 320 pp. (In Russian)

Received 06/IV/2015; received in revised form $17 / \mathrm{V} / 2015$; accepted 08/VIII/2015. 